\title{
Roadmap for a precision-medicine initiative in the Nordic region
}

\section{Njolstad, Pal Rasmus}

2019-06

Njolstad, P R, Andreassen, O A, Brunak, S, Borglum , A D , Dillner , J , Esko , T , Franks , P W , Freimer , N , Groop , L, Heimer , H, Hougaard, D M , Hovig , E , Hveem , K, Jalanko , A , Kaprio , J , Knudsen, G P , Melbye , M , Metspalu , A, Mortensen , P B , Palmgren , J, Palotie , A, Reed , W , Stefansson, H , Stitziel , N O , Sullivan , P F , Thorsteinsdottir , U , Vaudel , M , Vuorio , E , Werge , T , Stoltenberg , C \& Stefansson, K 2019 , ' Roadmap for a precision-medicine initiative in the Nordic region ' , Nature Genetics , vol. 51 , no. 6 , pp. 924-930 . https://doi.org/10.1038/s41588-019-0391-1

http://hdl.handle.net/10138/325612

https://doi.org/10.1038/s41588-019-0391-1

unspecified

acceptedVersion

Downloaded from Helda, University of Helsinki institutional repository.

This is an electronic reprint of the original article.

This reprint may differ from the original in pagination and typographic detail.

Please cite the original version. 


\section{Roadmap for a Precision Medicine Initiative in the 2 Nordic Region}

4 Pål Rasmus Njølstad $1,2,3^{*}$, Ole Andreas Andreassen ${ }^{4,5}$, Søren Brunak ${ }^{6}$, Anders

5 D. Børglum $7,8,9^{*}$, Joakim Dillner ${ }^{10,11}$, Tõnu Esko12,13, Paul W. Franks ${ }^{14,15,16,17^{*}}$,

6 Nelson Freimer ${ }^{18,19}$, Leif Groop ${ }^{14,20}$, Hakon Heimer ${ }^{21}$, David M.

7 Hougaard 22,23 , Eivind Hovig24,25,26, Kristian Hveem27,28,29, Anu Jalanko ${ }^{30}$,

8 Jaakko Kaprio ${ }^{20}$, Gun Peggy Knudsen ${ }^{31}$, Mads Melbye ${ }^{32,33,34}$, Andres

9 Metspalu ${ }^{35}$, Preben Bo Mortensen ${ }^{36,37}$, Juni Palmgren ${ }^{38,39}$, Aarno

10 Palotie $3,20,40,41,42,43^{*}$, Wenche Reed ${ }^{44}$, Hreinn Stefánsson45,46, Nathan 0.

11 Stitziel ${ }^{47,48,49}$, Patrick F. Sullivan ${ }^{50}$, Unnur Thorsteinsdóttir ${ }^{47,48}$, Marc

12 Vaudel $^{1}$, Eero Vuorio ${ }^{51}$, Thomas Werge ${ }^{52,53,22}$, Camilla Stoltenberg ${ }^{30,54^{*}}$ \&

13 Kári Stefánsson ${ }^{45,46}$

$15{ }^{1}$ Center for Diabetes Research, Department of Clinical Science, University of 16 Bergen, Bergen, Norway.

17 2Department of Pediatrics, Haukeland University Hospital, Bergen, Norway.

$18{ }^{3}$ Medical and Population Genetics Program, Broad Institute of Harvard and 19 MIT, Cambridge, MA,USA.

$20{ }^{4}$ NORMENT Centre, Division of Mental Health and Addiction, Oslo

21 University Hospital, Oslo, Norway.

22 5Institute of Clinical Medicine, University of Oslo, Oslo, Norway.

$23 \quad{ }^{6}$ Disease Systems Biology, Novo Nordisk Foundation Center for Protein

24 Research, Faculty of Health and Medical Sciences, University of

25 Copenhagen, Copenhagen, Denmark.

$26{ }^{7}$ Department of Biomedicine, Aarhus University, Aarhus, Denmark. 
27 8iSEQ, Centre for Integrative Sequencing, Aarhus University,

28 Aarhus, Denmark.

$29 \quad 9$ iPSYCH, Lundbeck Foundation Initiative for Integrative Psychiatric

30 Research, Aarhus, Denmark.

$31{ }^{10}$ Department of Laboratory Medicine, Karolinska Institutet, Stockholm,

32 Sweden.

$33{ }^{11}$ Karolinska University Laboratory, Karolinska University Hospital, 34 Stockholm, Sweden.

35 12The Estonian Genome Center, University of Tartu, Tartu, Estonia.

$36{ }^{14}$ Boston Children's Hospital, Harvard Medical School, Boston, MA, USA.

$37 \quad{ }^{14}$ Genetic and Molecular Epidemiology Unit, Lund University Diabetes

38 Centre, Lund University, Malmö, Sweden.

39 15Department of Public Health and Clinical Medicine, Section for Medicine,

40 Umeå University, Umeå, Sweden.

41 16Department of Nutrition, Harvard T. H. Chan School of Public Health, 42 Boston, MA, USA.

43 17Radcliffe Department of Medicine, University of Oxford, Oxford, UK.

44 18Semel Institute for Neuroscience and Human Behavior, Los Angeles, CA, 45 USA.

$46 \quad{ }^{19}$ UCLA Center for Neurobehavioral Genetics, Los Angeles, CA, USA.

$47 \quad$ 20FIMM Institute for Molecular Medicine Finland, University of Helsinki, 48 Helsinki, Finland.

49 21School of Health and Medical Sciences, University of Copenhagen, 50 Copenhagen, Denmark. 
51 22Department for Congenital Disorders, Statens Serum Institut,

52 Copenhagen, Denmark.

$53 \quad{ }^{23}$ iPSYCH - The Lundbeck Foundation Initiative for Psychiatric Research,

54 Copenhagen, Denmark.

$55{ }^{24}$ Department of Tumor Biology, Institute for Cancer Research, Oslo

56 University Hospital, Oslo, Norway.

$57 \quad 25$ Institute of Cancer Genetics and informatics. Oslo University Hospital,

58 Oslo, Norway.

59 26Department of Informatics, University of Oslo, Oslo, Norway.

60 27K.G. Jebsen Center for Genetic Epidemiology, Department of Public

61 Health, Norwegian University of Science and Technology, Trondheim,

62 Norway.

63 28HUNT Research Center, Department of Public Health, Norwegian

64 University of Science and Technology, Levanger, Norway.

$65{ }^{29}$ Department of Medicine, Levanger Hospital, Levanger, Norway.

$66{ }^{30}$ Genomics and Biomarkers Unit, National Institute for Health and Welfare,

67 Helsinki, Finland.

$68{ }^{31}$ Norwegian Institute of Public Health, Oslo, Norway.

69 32Department of Epidemiology Research, Statens Serum Institut,

70 Copenhagen, Denmark.

71 33Department of Medicine, Stanford University School of Medicine,

72 Stanford, CA, USA.

$73{ }^{34}$ Department of Clinical Medicine, University of Copenhagen, Copenhagen,

74 Denmark.

75 35The Estonian Genome Center, University of Tartu, Tartu, Estonia. 
${ }^{36}$ National Centre for Register-Based Research, Department of Economics

77 and Business Economics, Aarhus University, Aarhus, Denmark.

37iPSYCH - The Lundbeck Foundation Initiative for Psychiatric Research,

79 Aarhus, Denmark.

$80 \quad{ }^{38}$ Department of Medical Epidemiology and Biostatistics, Karolinska

81 Institutet, Stockholm, Sweden.

$82{ }^{39}$ Swedish e-Science Research Center, SeRC, Stockholm, Sweden.

83 40Psychiatric and Neurodevelopmental Genetics Unit, Massachusetts

84 General Hospital and Harvard Medical School, Boston, MA, USA.

$85 \quad{ }^{41}$ Stanley Center for Psychiatric Research, Broad Institute of MIT and

86 Harvard, Cambridge, MA, USA.

$87 \quad{ }^{42}$ Analytic and Translational Genetics Unit, Massachusetts General Hospital

88 and Harvard Medical School, Boston, MA, USA.

${ }^{43}$ Department of Neurology, Massachusetts General Hospital, Boston, MA,

90 USA.

$91 \quad{ }^{44}$ Department of Research, Innovation and Education, Oslo University

92 Hospital, Oslo, Norway.

$93 \quad{ }^{45}$ deCODE genetics, Reykjavik, Iceland.

$94 \quad$ 46University of Reykjavik, Iceland.

$95{ }^{47}$ Cardiovascular Division, Department of Medicine, Washington University,

96 Saint Louis, MO, USA.

$97 \quad{ }^{48}$ Department of Genetics, Washington University, Saint Louis, MO, USA.

$98{ }^{49}$ McDonnell Genome Institute, Washington University, Saint Louis, MO, 99 USA. 
100 50Department of Medical Epidemiology and Biostatistics, Karolinska

101 Institutet, Stockholm, Sweden.

102 51Department of Medical Biochemistry and Genetics, University of Turku,

103 Turku, Finland.

$104{ }^{52}$ Institute of Biological Psychiatry, Mental Health Center Sct. Hans, Mental

105 Health Services Capital Region of Denmark, Copenhagen, Denmark.

106 53Institute of Clinical Medicine, University of Copenhagen, Copenhagen,

107 Denmark.

$108{ }^{54}$ Department of Global Public Health and Primary Care, University of

109 Bergen, Bergen, Norway.

$110 *$ Members of the writing group

111

112 Address correspondence to: Professor Pål Rasmus Njølstad, M.D., Ph.D.,

113 Center for Diabetes Research, Hospital for Children and Adolescents,

114 Haukeland University Hospital, Haukelandsbakken 15, NO-5021 Bergen,

115 Norway (pal.njolstad@uib.no).

116

117

118 Abstract, 142 words; Text, 3,044 words; Figures, 1; Boxes, 3;

119 Supplementary Material, 1

120 


\section{Abstract}

122 The Nordic region, comprised here primarily of Denmark, Estonia, Finland, 123 Iceland, Norway, and Sweden, has many of the characteristics necessary to 124 be at the forefront of genome-based precision medicine. These include 125 egalitarian and universal health-care, expertly-curated patient and 126 population registries, biobanks, large population-based prospective 127 cohorts linked to registries and biobanks, and a widely embraced sense of 128 social responsibility that motivates public engagement in biomedical 129 research. However, this can only be achieved through coordinated action 130 involving all actors of the healthcare sector. Now is an opportune time to 131 organize scientists in the Nordic region, together with other stakeholders 132 including patient representatives, governments, pharmaceutical 133 companies, academic institutions, and funding agencies, to initiate a Nordic 134 Precision Medicine Initiative. We present a roadmap for how this can be 135 done. The Initiative will facilitate research, clinical trials, and knowledge 136 transfer to meet regional and global health challenges.

138 Background

139 Complex disease is caused by environmental exposures perturbing 140 multiple biochemical pathways. Determining the impact of these exposures 141 on the individual patient, and the extent to which these can be mitigated by 142 therapy, are fundamental objectives of contemporary medicine. One of the 143 major goals is to tailor medicine to the patient, not only by understanding 144 the disease, but also by understanding the specific characteristics of the 145 person. Another important goal is to accurately determine a person's risk 146 of developing disease, and to use this information to optimize the timing, 
147 delivery and type of preventive action. Collectively, these goals represent 148 precision medicine ${ }^{1}$ : individual preventive and therapeutic interventions 149 incorporating a detailed understanding of human diversity.

150 Human diversity is determined by the joint effects of environmental 151 exposures and DNA sequence variation². Genotyping millions of common 152 DNA variants in very large human populations has been made possible by 153 recent major technological advances. These technologies have been used to 154 discover and characterize many thousands of independent association 155 signals between common variants and disease traits. In general, common 156 single nucleotide polymorphisms (SNPs) individually confer relatively little 157 risk, but jointly, account for a substantial proportion of the overall 158 heritability of many complex disorders; for instance, in mental disorders, 159 the proportion of variance attributable to genome-wide SNPs (liability160 scale SNP heritability) is between a third and a quarter of the overall 161 heritability3,4. Methods have been developed to use a large number of 162 sequence variants to construct what is often termed a polygenic risk score 163 for phenotypes 5 . Polygenic risk scores can be useful in assessing both 164 relative and absolute risks of diseases. The frequencies and effects of 165 common variants tend to be similar in most ethnic groups with some 166 exceptions. The most recent breakthroughs in understanding the 167 relationships between human DNA sequence variation and phenotypic 168 diversity have been made possible by the development of technologies for 169 sequencing whole genomes or whole exomes in very large populations; this

170 in turn has identified rare risk variants, and expanded the catalogue of 171 verified polymorphic genomic regions related to disease ${ }^{6,7}$. Many of the 172 recently discovered rare variants cluster in the coding part of genes that 173 confer high risk of various diseases ${ }^{8}$. It is, however, important to remember 174 that the rare variants have arisen recently and tend to be population-, or 
175 even family-, specific. Nevertheless, even de novo mutations, affecting the

176 probands but not their parents, have proven relevant for public health ${ }^{9,10}$.

177 Although these major technical advances in human genetics have been a 178 driving force in the evolution of precision medicine, there are many 179 practical obstacles hindering the implementation of these technologies at 180 scale. We argue that the Nordic countries (Fig. 1) offer a priviledged 181 environment to negotiate these obstacles making it likely that, with a well182 functioning organizational structure and adequate funding, the Nordic 183 countries will likely play a very significant role in precision medicine over 184 the coming years, at least as it relates to the diseases common within the 185 region. Indeed, given the region's unique resources, we feel obligated to 186 ensure that this possibility is realized.

187 Although major progress has been made in disease genomics, further 188 characterization will require very large prospective cohorts with 189 harmonized genetic and phenotypic data, yet such datasets are uncommon 190 in most parts of the world. Over recent decades, the Nordic countries have 191 individually collected very large, carefully phenotyped, prospective 192 cohorts, often for the sake of disease monitoring and surveillance. These 193 cohorts and registries contain data from the majority of Nordic citizens, 194 following many of them throughout parts of the life-course; data are often 195 collected through primary care clinics, hospitals, and post-mortems. This 196 process has resulted in the availability of datasets comprising some 27 197 million Nordic citizens (Fig. 1), many of whom have undergone repeated 198 sampling over many decades. Notably, a considerable amount of today's 199 knowledge on the epidemiology of human disease is based on research 200 from Nordic cohorts and registries (Supplementary Table 1). 
201 The Nordic countries include Denmark, Finland, Iceland, Norway, and 202 Sweden, with their associated territories (Greenland, the Faroe Islands, and 203 the Åland Islands). The 27 million people of the Nordic countries are 204 ancestrally mainly Scandinavian or Finnish, with Greenlandic Inuit (around 205 56,000) and the Sami $(50-80,000)$ as indigenous peoples. Estonians share 206 language and historical roots with the Finns and identify very closely with 207 Nordic culture. Furthermore, with its well-established national biobank, 208 Estonia has been an active partner in the Nordic Biobank Network. Despite 209 the fact that the region for centuries has had immigration from 210 neighboring, continental countries, and more recently from elsewhere, 211 immigrants still comprise only a minor proportion of the Nordic 212 population. In 2012 for instance, 10.9\%, 7.9\%, and 14.9\% of the 213 Norwegian, Danish, and Swedish populations were immigrants, 214 respectively (Statistics Norway). The Nordic countries have much in 215 common in their way of life, history, social structures, and languages. These 216 countries do not form a truly united political entity, but co-operate closely 217 on many levels, including within the Nordic Council.

219 Access to unique data needed to apply precision medicine

220 One of the assets needed for the implementation of precision medicine is 221 collections of data on individual phenotypes and genotypes that allow 222 detailed studies of the causal effects of genetic variants in disease. Such 223 datasets can be used for the crucial replication of published genetic 224 associations with disease and other relevant phenotypes, as well as for 225 determining population-specific frequencies and the extent to which 226 specific variants impact disease (effect size or predictive accuracy). These 227 datasets are, however, of greatest value for i) discovering genomic variants 
228 that pinpoint druggable pathways, ii) aiding in the reclassification of

229 disease diagnoses (to generate new taxonomies that can be treated more 230 effectively), and iii) facilitating the stratification of populations based on 231 risk factor susceptibility or therapuetic response (to optimize prevention 232 or treatment), each of which are core features of precision medicine.

233 Although today there is no truly pan-Nordic database on phenotypes and 234 genotypes, the required components exist. These include: (a) a long history 235 of integrated healthcare, patient registries and biobanks, with existing 236 assets of biological samples, patient records, and longitudinal follow-up;

237 (b) population characteristics such as founder effects and stable, traditional 238 societies with homogeneous environmental exposures; (c) strong public 239 trust based on a history of social welfare and commitment to research for 240 the public good; and (d) access to technology and expertise for generating, 241 managing, storing and interpreting genomic and clinical data 242 (Supplementary Table 2). What remains is to bring together the wealth of 243 data and biomaterials under a common framework, and to make this 244 accessible to the research community through federated data-access 245 models.

246 No other countries currently have access to population-based registries of 247 comparable size and with similar quality and detail of clinical information 248 as those of the Nordic nations (Box 1). The unique features of this Nordic 249 resource include complete nationwide social and health registers from 250 about 1950 onwards, the world's largest health studies with detailed 251 phenotypes, biological samples, follow-up of 30-50 years, hospital 252 diagnoses as well as prescription and treatment registries, including all 253 inpatients and outpatients for all hospitals during the past decades, and 254 newborn screening programs of live births with samples stored since the 255 early 1980s. There are population-based biorepositories and data from at 
256 least 8-10 million individuals available for research in the Nordic countries

257 today.

258 Regarding the secure storage and use of data and the challenge of utilizing 259 data while protecting the data donor's privacy, the Nordic region has 260 advantages due to its traditions of equality and a strong public sector. The 261 citizens generally commit to studies with broad consent. In all Nordic 262 countries, there is an overall positive attitude toward health research, 263 including genetic studies ${ }^{11}$. There is a healthy balance between privacy 264 regulations and willingness to share data for research. New European 265 Union regulations may, however, create new challenges (Box 2). As the 266 European General Data Protection Regulation (GDPR) came into effect in 267 May 2018, its full consequences remain unknown. There are, however, 268 clear ways in which GDPR expectations can be fulfilled. Implied consent is 269 sufficient for data that are not sensitive, while analysis of sensitive data 270 requires opt-in consents specific for each research question addressed with 271 the data.

272 deCODE genetics in Iceland and through its collaboration elsewhere in the 273 Nordic region has been at the forefront of human genomic research for two 274 decades (Supplementary Table 1). This engagement corresponds with the generation of extensive genotype data from SNP arrays for some 650,000

276 Nordic participants. Whole exome or genome sequence data from over 27795,000 Nordic participants have also been generated. deCODE genetics has 278 genotyped half of the Icelandic nation and performed whole genome 279 sequencing on ten percent of $i^{12}$. In addition to work done in the Nordic 280 countries ${ }^{13}$, recent significant contributions to human genetics coming 281 from the USA have been made through the use of clinical material, 282 registers, cohorts, and biobanks in the Nordic countries (Supplementary 283 Table 1$)^{14,15}$. A pilot project on colorectal cancer supported by the Nordic 
284 Council of Ministers has connected biobanks and registries in all Nordic 285 countries, including transfer of personal data between Nordic countries as 286 well as joint genotyping and whole genome sequencing of biospecimens 287 from several Nordic countries, demonstrating that the infrastructure and 288 regulations allow considering the Nordic countries as a single scientific 289 region (NordForsk).

290 The Nordic countries have also been at the forefront of epidemiological 291 studies linking environmental exposures to disease outcomes. These 292 studies have identified the diversity in exposure to important external 293 determinants of disease. Until now, these have been largely independent of 294 genetic information, and genetic association studies have mostly been 295 conducted independently of known environmental risk factors, despite many large-scale epidemiological studies in the Nordic countries during the past 50 years. Whilst the origins of precision medicine have come from 298 population genetics research, the successful implementation of precision medicine to tackle common complex disease will almost certainly require consideration of the joint effects of genes and environment, as lifestyle has a major influence on most common diseases. Recently, DNA has been 302 extracted, genotyped or sequenced in many of these outstanding Nordic cohorts, enabling adequately powered pioneer studies uncovering the interplay of the environment and genetics.

A special feature of the Nordic countries is the existence of multiple genetic 306 isolates, some of which are contained within the large registers and

307 biobanks. This unique resource has facilitated the discovery of variants that are private to specific population isolates or families. An example is detection of a rare loss-of-function (LoF) variant in the SLC30A8 gene that

310 is protective for type 2 diabetes and enriched in the Botnia region ${ }^{14}$. To 311 explore the underlying mechanisms, we were able to go back to the families 
312 carrying the LoF mutation and by sequencing additional family members,

313 increased the number of mutation carriers 3-fold. We then selectively

314 recruited participants by genotype for additional metabolic studies to 315 pinpoint the mechanisms for protection. Although Finland is not technically 316 an isolate, its history - small founder population, evolutionary bottleneck

317 and then rapid expansion - makes it ideal for identifying rare mutations.

318 Several initiatives that pave the way for a Nordic precision initiative are 319 now underway in individual countries: The Danish government and 320 municipal authorities have recently launched a national project in precision 321 medicine for the use of genetic analysis technologies in the prevention and 322 treatment of many diseases. Novo Nordisk Foundation has recently 323 announced its support this initiative with over 900 mill DKK (approx. 137 324 mill USD). In Finland, major changes in the legal framework are currently 325 undergoing parliament hearings: National registers, genome, and biobank 326 legislation will be reorganized to improve the secondary use of health data 327 in research and development. The Research Council of Norway, as well as 328 major universities and university hospitals, and the Norwegian Institute of 329 Public Health, are increasingly supporting projects on precision medicine, 330 and the legal framework is becoming more positive towards genetic studies 331 utilizing population registers and biobanks. The Swedish government has 332 commissioned the Swedish Research Council to support national 333 infrastructure for register-based research, including clinical registers. 334 Work is ongoing to create a single national entry point for research using 335 registers, cohorts and biobanks. The NordForsk funding agency is further 336 actively supporting development of mechanisms for secure sharing of 337 person-sensitive data across the Nordic countries through Tryggve, and the 338 Nordic ELIXIR nodes are similarly enabling secure data exchange through 339 participation in national European Phenome-Genome repositories. 
340

341

342

343

344

345

346

347

348

349

350

351

352

353

354

355

356

357

358

359

360

361

362

363

364 365

SNP array studies have shown a close correspondence between genetic and geographic distances in Europe and that the geographical map of Europe naturally arises as an efficient two-dimensional summary of genetic variation in Europeans ${ }^{16,17}$. Their descent can genetically, and hence geographically, be distinguished by drawing a line from the north to the south-east (northern Europe to the Balkans), with another east-west axis across Europe. Y-chromosome studies show three large haplogroups that account for most of Europe's patrilineal descent. Nordic populations overlap considerably, particularly in major cities and neighboring regions, but differ from other European populations in their genetic substructure, with Finns being especially distinct, to the extent that they are essentially a genetic isolate ${ }^{18}$. This is also reflected in the language groups (Supplementary Fig. 1). Hence, in addition to the wisdom of working with the biobanks in the individual Nordic populations and the ability to link them to population registries on healthcare information and other relevant demographic data, the shared ancestry of the Nordic peoples that is reflected in overlap of genomic sequences makes them ideal partners in genetic research and in the implementation of precision medicine (Supplementary Fig. 2).

\section{Healthcare with universal access and societal acceptance}

Another asset necessary for early implementation of precision medicine is government-funded healthcare systems with excellent records and universal access that are focused on longterm benefits to society rather than shortterm profit. An additional asset is having societies that are committed to protecting the rights of individuals to privacy while 
366 recognizing the importance of using healthcare information for discoveries

367 that improve health and care.

368 A number of factors predict that precision medicine may be implemented 369 across the region without worsening health disparities. Indeed, we argue 370 that this strategy is a necessary extension of a number of economic and 371 cultural specialities of the region. The Nordic countries rank at the top in a 372 range of metrics of national economic performance, including education, 373 digitalization, economic competitiveness, civil liberties, quality of life, and 374 human development ${ }^{19}$. Together, the economies of the Nordic countries 375 have one of the best macroeconomic performances in the world, and are 376 leaders in sustainable development. The Nordic countries also share many 377 aspects of their economic systems and social structures: market economy is 378 combined with relatively strong labor unions and a well-developed public 379 welfare sector ${ }^{20}$. There is a high degree of income distribution and 380 relatively little social unrest. Individual rights are secured legally and have 381 an increasingly strong influence on the health care systems. In general, 382 inhabitants of the Nordic countries are positive towards research and 383 frequently consent to genetic research with a wide scope both as 384 participants in disease-specific cohorts and population-based general 385 health surveys. The Nordic countries all have single payer healthcare 386 systems with good access and quality of services. Our precision medicine 387 strategy hence appears as a natural extension of economic and cultural 388 specificities of the region.

390 In spite of these qualities of the Nordic societies, extensive coordination 391 and ambitious funding strategies are required to achieve the necessary 392 societal support to enable the implementation of precision medicine.

393 Efforts to introduce population genomics in the Nordic countries will rely 
394 on a combination of public and foundation funds, as well as investments 395 from biotechnology and pharmaceutical industries. This can induce 396 concerns about the protection of the rights and privacy of citizens, which 397 will need to be adequately addressed if public support is to be maintained. 398 The European GDPR offers the opportunity for the Nordic countries to align 399 processes for personal data used in research. A second issue is the need to 400 find solutions to how the value that is generated in the international 401 private sector using samples and data from the Nordic region shall be 402 returned to the nations and citizens who funded and generated these 403 resources. A third good example of the opportunities and challenges ahead 404 is provided by the story of the BRCA2 mutation in Iceland. Work done at 405 deCODE genetics has brought insight into the whole genomes of most of the 406 nation. Hence, all BRCA2 mutation carriers in Iceland could in theory be 407 identified in silico and offered interventions that mitigate the cancer risk 408 conferred by the mutation ${ }^{21,22}$. This would be an excellent example of how 409 precision medicine can contribute substantially to public health. This has,

410 however, not been done yet, because the Icelandic society is still debating 411 how to approach the mutation carriers with this clinically critical 412 information. There are people who are deeply concerned about the right of 413 the carriers not to know about their genetic risk. Shall the participants in 414 research studies always have the right to learn the results, even if the 415 medical consequences of the genetic discovery are not yet fully 416 understood? As this situation illustrates, the obstacles to the 417 implementation of precision medicine are not only financial, technical and 418 scientific, but also societal and ethical. We believe that the people of the 419 Nordic region are ready to tackle these challenges and offer a positive 420 example for the rest of the world ${ }^{11}$. 


\section{A roadmap for the way forward}

423 Recently, the Nordic Society for Human Genetics and Precision Medicine 424 was formed and launched a roadmap for the way forward (Box 3). The 425 Society was created in order to: (a) accelerate discovery of disease 426 susceptibility genes and genes protecting from disease through integrated 427 analyses using multiple large-scale datasets and a range of experimental 428 designs; (b) translate these findings so that they can be used for precision 429 medicine to improve public health; (c) and uphold and promote the highest 430 legal, regulatory, social, and ethical standards.

431 The Society will also be a vehicle to engage the many constituencies of 432 precision medicine, ranging from research and clinical geneticists to data 433 scientists and legal experts. It will also allow for the communication of 434 accurate, up-to-date information to policymakers, research funders, and, 435 most importantly, the public.

436 We believe our initiative will accelerate research, clinical trials, and 437 transmission of knowledge to meet numerous local, regional, and global 438 health challenges, taking advantage of the unique Nordic health-care 439 system, patient and population registries and biobanks, as well as the social 440 responsibility that has motivated public engagement in biomedical 441 research.

443 URLS

444 Danish strategy for personalized medicine, http://www.sum.dk/English.aspx;

445 deCODE genetics, https://en.wikipedia.org/wiki/DeCODE genetics;

446 European Commission, Joint Research Center, http://data.europa.eu/89h/jrc- 
447 ghsl-ghs pop gpw4 globe r2015a; genotyping in Nordic countries,

448 https://www.nordforsk.org; Nordic Council of Ministers pilot project on

449 colorectal cancer, https://www.nordforsk.org/en/policy/policy-briefs-1;

450 Nordic countries, https://en.wikipedia.org/wiki/Nordic countries; Nordic

451 Society for Precision Medicine, http://nordicprecisionmedicine.org/;

452 Nordic platform for collaboration on sensitive data,

453 https://wiki.neic.no/wiki/Tryggve; Nordic region and sustainable

454 development, http://blogs.worldbank.org/governance/among-wealthy-

455 nations-nordic-countries-are-leading-pack-sustainable-development;

456 publications from deCODE genetics,

457 https://www.decode.com/publications/; Statistics Norway,

458 https://www.ssb.no/en/befolkning

459

460 Acknowledgements

461 We thank the Banbury Center at Cold Spring Harbor Laboratory, New York

462 for organizing and hosting the meeting "Studying the Genomic Variation

463 that Underlies Health and Disease: The Unique Contribution of the Nordic

464 Health Systems" at the Banbury Center February 16-19, 2016. We are

465 grateful to Drs. C.E. Jaquish, G. Tybring as well as D. Høybråten for helpful

466 discussions. We acknowledge funding from the Norwegian Research

467 Council (\#223273 to O.A.A.) and NordForsk (to P.W.F.). The work 468 conducted by P.W.F. that is germane to this paper was supported by the 469 Swedish Research Council and ERC-2015-CoG_NASCENT_681742. P.R.N.

470 was supported by grants from the European Research Council (AdG 471 \#293574), the Research Council of Norway (\#240413), Helse Vest 472 (PERSON-MED_DIA), and Stiftelsen Kristian Gerhard Jebsen (Center for 473 Diabetes Research). 
476 P.R.N., P.W.F., A.D.B., A.P., C.S, and K.S. wrote the manuscript with edits from 477 O.A.A., S.B., J.D., T.E., N.F., L.G., H.H., D.M.H., E.H., K.H., J.K., G.P.K., M.M., 478 A.M., P.B.M., J.P., A.P., W.R., H.S., N.O.S., P.F.S., U.T., M.V., E.V., and T.W. 479 P.R.N., O.A.A., S.B., A.D.B., J.D., T.E., P.W.F., N.F., L.G., H.H., D.M.H., E.H., K.H., 480 A.J., J.K., G.P.K., M.M., A.M., P.B.M., J.P., A.P., W.R., H.S., N.O.S., P.F.S., U.T., 481 M.V., E.V., T.W., C.S., and K.S. conceived the project.

482

483 Competing financial interests

484 H.S., U.T., and K.S. are employed by deCODE Genetics/Amgen, Inc. The other 485 authors declare no competing financial interests. 
487 1. National Research Council (US) Committee on A Framework for 488 Developing a New Taxonomy of Disease. Toward precision medicine: 489 building a knowledge network for biomedical research and a new 490 taxonomy of disease. National Academies Press, Washington, DC, USA, 4912011.

492 2. Collins, F.S., Green, E.D., Guttmacher, A.E., Guyer, M.S., US National 493 Human Genome Research Institute. A vision for the future of genomics 494 research. Nature 422, 835-847 (2003).

495 3. Cross-Disorder Group of the Psychiatric Genomics Consortium. Genetic 496 relationship between five psychiatric disorders estimated from genome497 wide SNPs. Nat. Genet. 45, 984-994 (2013).

498 4. Schizophrenia Working Group of the Psychiatric Genomics Consortium. 499 Biological insights from 108 schizophrenia-associated genetic loci. Nature 500 511, 421-427(2014).

501 5. Khera, A.V. et al. Genetic risk, adherence to a healthy lifestyle, and 502 coronary disease. N. Engl. J. Med. 375, 2349-2358 (2016).

503 6. Gudbjartsson, D.F. et al. Large-scale whole-genome sequencing of the 504 Icelandic population. Nat. Genet. 2015 May;47(5):435-44.

505 7. Marouli, E. et al. Rare and low-frequency coding variants alter human 506 adult height. Nature 542, 186-190 (2017).

507 8. Lek, M. et al. Analysis of protein-coding genetic variation in 60,706 508 humans. Nature 536, 285-291 (2016).

509 9. Acuna-Hidalgo, R., Veltman, J.A., Hoischen, A. New insights into the 510 generation and role of de novo mutations in health and disease. Genome 
511 Biol. 17, 241 (2016).

512 10. Kong, A. et al. Rate of de novo mutations and the importance of father's 513 age to disease risk. Nature 488, 471-475 (2012).

514 11. Ebbesen, M., Sundby A, Pedersen FS, Andersen S. A philosophical 515 analysis of informed consent for whole genome sequencing in biobank 516 research by use of Beauchamp and Childress' four principles of biomedical 517 ethics. J. Clin. Res. Bioeth. 6, 244 (2015).

518 12. Editorial. Letters from Iceland. Nat. Genet. 47, 425 (2015).

519 13. Maretty, L. et al. Sequencing and de novo assembly of 150 genomes 520 from Denmark as a population reference. Nature in press (2017).

521 14. Flannick, J. et al. Loss-of-function mutations in SLC30A8 protect against 522 type 2 diabetes. Nat. Genet. 46, 357-63 (2014).

523 15. Fuchsberger, C. et al. The genetic architecture of type 2 diabetes. Nature 524 536, 41-47 (2016).

525 16. Novembre, J., et al. Genes mirror geography within Europe. Nature 456, 526 98-101 (2008).

527 17. Palo, J.U., Ulmanen, I., Lukka, M., Ellonen, P., Sajantila, A. Genetic 528 markers and population history: Finland revisited. Eur. J. Hum. Genet. 17, 529 1336-1346 (2009).

530 18. Lao, O. et al. Correlation between genetic and geographic structure in 531 Europe. Curr. Biol. 18, 1241-1248 (2008).

532 19. Nordic Statistical Yearbook 2014 (ed. Haagensen, K.M.). Nordic Council 533 of Ministers, 52, 7-135. Nord, Copenhagen, 2014 (ISBN 978-92-893-3854534 7).

535 20. Wooldridge, A. Northern lights. Special report: the Nordic countries. 
536 The Economist Henry Thoreau and Enterprise 2, 1-14 (2013).

537 21. Thorlacius, S. et al. A single BRCA2 mutation in male and female breast

538 cancer families from Iceland with varied cancer phenotypes. Nat. Genet. 13, 539 117-119(1996).

540 22. Thorlacius, S. et al. Study of a single BRCA2 mutation with high carrier

541 frequency in a small population. Am. J. Hum. Genet. 60, 1079-1084 (1997).

542 23. Deloukas, P. et al. Large-scale association analysis identifies new risk

543 loci for coronary artery disease. Nat. Genet. 45, 25-22 (2013).

544 24. Ripke, S. et al. Genome-wide association analysis identifies 13 new risk

545 loci for schizophrenia. Nat. Genet. 45, 1150-1159 (2013).

546 25. Fuchsberger, C. et al. The genetic architecture of type 2 diabetes.

547 Nature. 536, 41-47 (2016).

548 26. Willer, C.J. et al. Discovery and refinement of loci associated with lipid

549 levels. Nat. Genet. 45, 1274-1283 (2013).

550 27. Melin, B.S. et al. Genome-wide association study of glioma subtypes

551 identifies specific differences in genetic susceptibility to glioblastoma and

552 non-glioblastoma tumors. Nat. Genet. 49, 789-794 (2017).

553 28. Lambert, C.J. et al. Meta-analysis of 74,046 individuals identifies 11 new

554 susceptibility loci for Alzheimer's disease. Nat. Genet. 45, 1452-1458

555 (2013) 29. Law, M.H. et al. Genome-wide meta-analysis identifies five new 556 susceptibility loci for cutaneous malignant melanoma. Nat. Genet. 47, 987557995 (2015).

558 
559 Figure legend

560 Figure 1: The Nordic countries, a geographical and cultural region in 561 Northern Europe and the north Atlantic sea.

562 The Nordic region has around 27 million inhabitants. The population 563 number is shown for Iceland, the kingdoms of Denmark, Norway, and 564 Sweden, and the republic of Finland, and associated territories. These are 565 Greenland and Faroe Islands (ruled by Denmark), Åland Islands (ruled by 566 Finland), and Svalbard (ruled by Norway). Estonia is often associated with 567 the Nordic countries as well. Population density as of 2015 was obtained 568 from the Global Human Settlement project and displayed in shades of grey 569 (Supplementary Note; European Commission, Joint Research Centre, 570 Columbia University, NYC, NY). Abbreviations: k, thousand; mil, million; 571 inhab, inhabitant.

572

573 
Box 1: Selected examples of population-based cohorts, biobanks and registers in the Nordic region

Denmark

The Danish National Biobank at Statens Serum Institut (www.ssi.dk) contains eight million specimens increasing by 0.5 million annually. Most are serum, plasma and DNA, although there also are spinal fluids, faeces and urine. Eleven robots are effectively performing pipetting, DNA purification, biomarker analysis, and storage. Detailed phenotypic data can be obtained via a linkage to national registers.

The National Biobank Register (www.nationalbiobank.dk) includes 22 million specimens from 5.4 million persons in Danish biobanks. Combining data with register information allows searching for diagnosis, age, sex and other variables as well as specific searches that e.g. will identify specimens taken at specific time points before, at, or after a certain diagnosis. Participation and use is free of charge.

Bio- and Genome Bank Denmark (www.regioner.dk/rbgben) is the entry to biological material at all hospitals. The material is professionally collected, nationally registered and regionally stored in five hospitals. Pathological material is stored locally for clinical use. Phenotypic data can be obtained via linkage to registers and clinical databases.

The Danish Neonatal Screening Biobank includes filter blood spots from two million subjects, almost all that are born since 1982. iPSYCH (www.ipsych.au.dk) is the largest study utilizing these samples, including GWAS of 80,000 individuals, of which 50,000 suffer from mental disorders, integrating genomic and national register data.

The Danish National Birth Cohort (www.ssi.dk) contains holds questionnaire data from pregnancy $(100,000)$ and the offspring at six and 18 months as well as seven and 11-year follow-ups. Biological specimens have been collected twice during pregnancy together with cord blood (Danish National Biobank).

Estonia

The Estonian Biobank is population-based (www.biobank.ee) including medical history and current health status as well as extra data from psychiatric patients. All samples $(155,000)$ have been genotyped (SNP arrays); while exome sequencing has been done on 2,500 and whole 
genome sequence on 2,500 samples. The biobank can be linked with national health registries and hospital databases.

Finland

The National Institute of Health and Welfare (THL) hosts the majority of large epidemiological and disease specific cohorts (e.g. Finrisk, Health2000, Twins, Botnia, Migraine, GeneRisk) that contain blood samples from 200,000 individuals (www.thl.fi/biobank) of all ages. These include questionnaire, genomic, and biochemical data and link to National Health Register data (EHR) providing decades of disease follow-up data. All university hospitals have established biobanks linking sample data with EHR and EMR data. Hospital biobanks host tissue samples from almost 3 million participants, collected earlier as part of routine diagnostics and have recently been transferred to biobanks. The hospital biobanks as well as the Blood Service Biobank have recently collected prospective samples from 120,000 participants and continue rapidly expanding their collections. The rapid expansion is due Finnish Biobanks partnering with the public-private FinnGen project aiming to collect GWAs and national health register data from 500,000 participants by 2022 (https://www.finngen.fi/). The FinnGen research project has been a major facilitator for development of national biobanking and the accumulated genome data of FinnGen currently 145000 - is foreseen to serve as a major basis for GWAS and PheWAS analyses towards development of personalized medicine. For biobanks, the newly produced genome data will further enrich the EHR data with symptom-level information, pathology and biochemical data thus building more possibilities for excellent science and development. All biobanks are networked by BBMRI.fi and have harmonized broad consents and practices.

Iceland

deCODE genetics (www.decode.com) has gathered genotypic and medical data from over 160,000 participants, well over half of the adult population. Using Iceland's uniquely comprehensive genealogical records, deCODE has also a genealogy database covering the entire present day population stretching back to the founding of the country more than 1,000 years ago. The combination of size of the population, the participation of so many people in the discovery work, the genealogies, and high quality universal healthcare have made possible very large-scale studies of virtually any common disease. At the same time, deCODE's work has minimized the selection bias that confronts research in larger, more stratified populations, enabling to impute or predict genotypes using the genealogies, multiplying many-fold the 
amount of data that can derive from genotyping and sequencing.

Norway

The Norwegian Mother and Child Cohort Study (MoBa) is a pregnancy cohort $(114,500$ children, 95,000 mothers, 75,000 fathers) recruited 1999-2009 (www.fhi.no/MoBa). Information on health and exposures are collected from questionnaires during pregnancy and regularly after birth, and by linkage to registries. Biomaterials were collected from fathers and mothers at pregnancy week 17 and after birth, and from umbilical veins. DNA has been extracted. 25,000 triads have been genotyped (SNP arrays). Many outcome registries have been linked to the cohort.

The Health Survey of Northern Trøndelag (HUNT) contains medical histories and specimens $(120,000)$ from a homogeneous population collected over 30 years (www.hunt.no). Three surveys include information on health-related lifestyle, prevalence and incidence of diseases, health determinants, and associations between disease phenotypes and genotypes. 70,000 samples have been genotyped (SNP arrays). Data can be linked to national health registries.

The Troms $\varnothing$ Study is prospective and population-based with six repeated health surveys after 1974 (www.tromsoundersokelsen.no) including questionnaire data, DNA, serum, and clinical measurements. 40,000 subjects attended at least once, and 15,000 in three surveys or more. In addition to national quality controlled disease registries, the study holds a validated endpoint registry of many well-defined diseases.

The Hordaland Health Studies (husk-en.b.uib.no) were conducted in 1992/93 (The Homocysteine study) and in 1997/99 (HUSK). The main focus is cardiovascular disease, cancer, osteoporosis, anxiety and depression. Some 36,000 residents of Hordaland county participated; 18,000 in $1992 / 93$ and 26,000 in $1997 / 99$. About 7,000 of those who participated in the 1992/93 survey also participated in 1997/99.

Sweden

The Genomic Aggregation Project in Sweden (GAPS) has around 30 Swedish cohorts within which existing detailed genetic and phenotypic data is available $(170,000)$. Within these cohorts exists tens of thousands of additional data-points against which blood samples are stored, from which DNA will be extracted for future genotyping and sequencing. The cohorts already genotyped include the Malmö Diet and Cancer cohort $(28,000)$ in southern Sweden, the Breast Cancer Studies 
cohort $(30,000)$ in the central Sweden, the GLACIER Study $(20,000)$ in northern Sweden, and multiple case-control and cohort studies of severe psychiatric disorders (63,000). Aside from disease record linkage, cohorts in Sweden are frequently linked to the drug registry and demographic databases (allowing genealogies dating back to the 1700 s to be linked with genetic and phenotypic data).

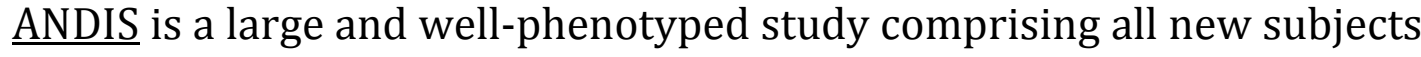
with diabetes in Skåne, a complete-capture case series of $>15,000$ patients with diabetes, from which samples have been genotyped and linked to a range of cross-sectional and prospective registry databases.

Text box 2: The European Union's General Data Protection Regulation and the Nordic Countries

The General Data Protection Regulation (GDPR, Regulation (EU) 2016/679) of May 25, 2018 replaced the Data Protection Directive (officially Directive 95/46/EC on the protection of individuals with regard to the processing of personal data and on the free movement of such data). The European Commission's (EC) objectives with this new legislation included the harmonization of 27 national data protection regulations into one unified regulation, the improvement of corporate data transfer rules outside the European Union (EU), and the improvement of user control over personal identifying data. The proposed new EU data protection regime thus extends the scope of the EU data protection law to all foreign companies processing data of EU residents. It provides for a harmonization of the data protection regulations throughout the EU, thereby making it easier for nonEuropean companies to comply with these regulations; however, this comes at the cost of a strict data protection compliance regime with severe economic penalties.

The GDPR preserves the equilibrium between the necessity of effectively protecting the subject's rights in a digitalised and globalized world while allowing the processing of personal data, including sensitive data, for scientific research. It reinforces cooperation duties and transparency between the actors of the processing, internally and with regard to the supervisory authorities, which should create a more integrated EU data protection system and diminish some useless administrative costs by decentralising elements of the data protection governance towards data controllers and processors. Whilst the GDPR adopts new specific provisions to ensure adapted data protection in research, the field remains widely regulated at national level, in particular, regarding the application of research participants' rights, 
which some could regret. However, the GDPR has the merit to set up clearer rules that will positively serve the research practices notably regarding consent, regarding the rules for reusing personal data for another purpose, assessing the risks of data processing in the context of data protection impact assessment, adopting accountable management system of processing operations and building or reinforcing internal data protection competencies with the data protection officer. In addition, for the first time, the GDPR refers to the respect of ethical standards as being part of the lawfulness of the processing in research, what must be welcomed as an effort for sector-specific consistency. Finally, the GDPR opens new possibilities for structuring data sharing in scientific research with measures encouraging self-regulation development.

Box 3: Roadmap for the precision medicine initiative in the Nordic region

Develop the Nordic Society for Human Genetics and Precision Medicine, among whose tasks will be to:

- Organize biannual large open scientific meetings

- Organize a series of workshops targeted to the constituencies of precision medicine, e.g., research geneticists, clinical geneticists, data scientists, legal scholars

- Write a white paper that summarizes the major needs

- Develop web-based resources, including a news feed, continuous updated overview of available cohorts, registers, and biobanks, as well as linked genomics and metabolomics information

Engage with important constituencies

- Policymakers

- The public

- Other organizations in this sphere

- Interact with funding partners

- NordForsk

- National research councils

- Private non-profit organizations and foundations

- Industry 


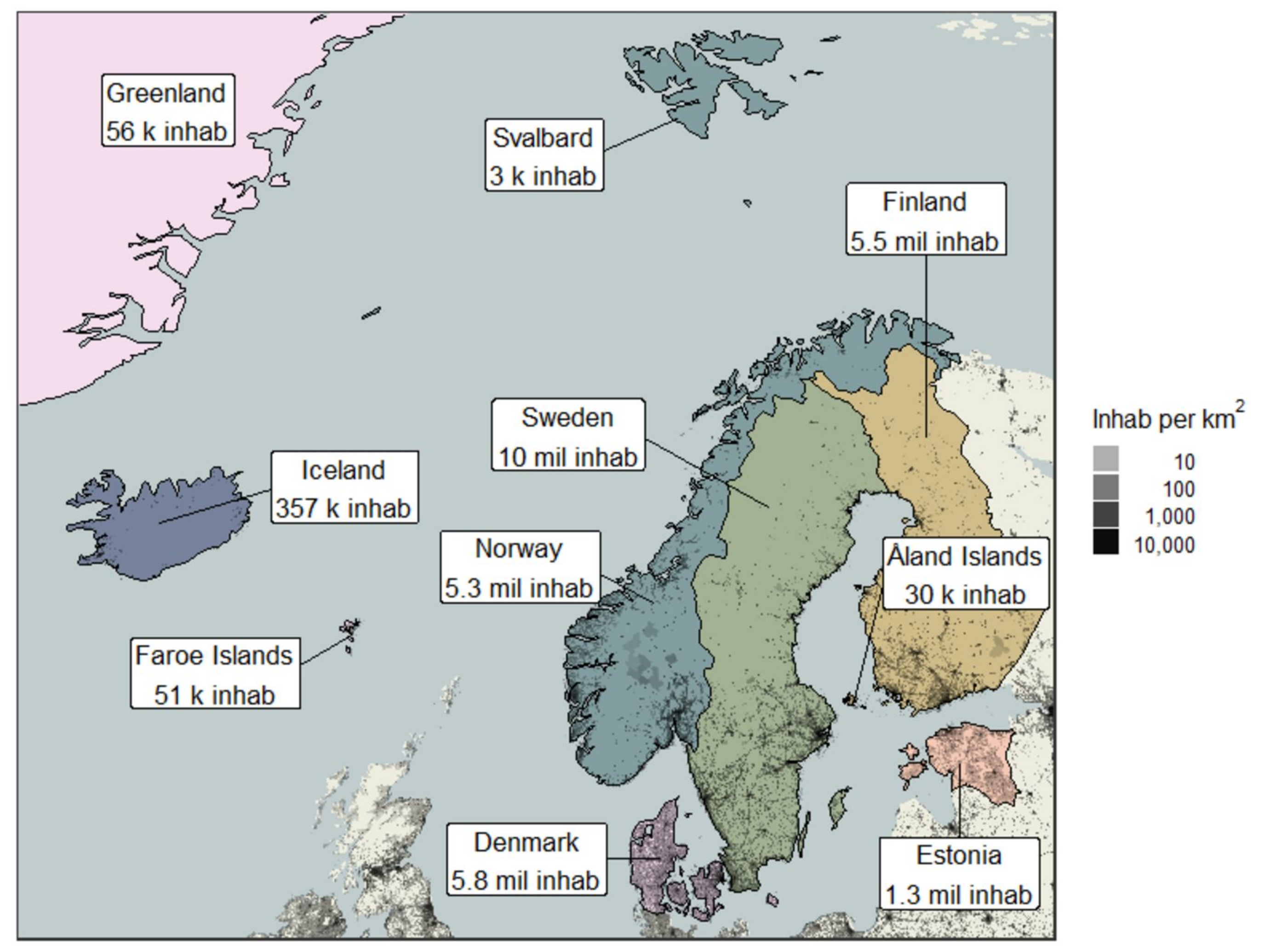




\section{Roadmap for a Precision Medicine Initiative in the Nordic Region}

Pål Rasmus Njølstad $1,2,3^{*}$, Ole Andreas Andreassen ${ }^{4,5}$, Søren Brunak ${ }^{6}$, Anders D. Børglum ${ }^{7,8,9^{*}}$, Joakim Dillner ${ }^{10,11}$, Tõnu Esko12,13, Paul W. Franks ${ }^{14,15,16,17^{*}}$, Nelson Freimer $^{18,19}$, Leif Groop ${ }^{14,20}$, Hakon Heimer ${ }^{21}$, David M. Hougaard ${ }^{22,23}$, Eivind Hovig 24,25,26, Kristian Hveem ${ }^{27,28,29}$, Anu Jalanko ${ }^{30}$, Jaakko Kaprio ${ }^{20}$, Gun Peggy Knudsen ${ }^{31}$, Mads Melbye $^{32,33,34}$, Andres Metspalu ${ }^{35}$, Preben Bo Mortensen ${ }^{36,37}$, Juni Palmgren ${ }^{38,39}$, Aarno Palotie $3,20,40,41,42,43^{*}$, Wenche Reed ${ }^{44}$, Hreinn Stefánsson ${ }^{45,46}$, Nathan O. Stitziel $47,48,49$, Patrick F. Sullivan ${ }^{50}$, Unnur Thorsteinsdóttir ${ }^{47,48}$, Marc Vaudel ${ }^{1}$, Eero Vuorio ${ }^{51}$, Thomas Werge $^{52,53,22}$, Camilla Stoltenberg ${ }^{30,54^{*}}$ \& Kári Stefánsson ${ }^{45,46}$

${ }^{1}$ Center for Diabetes Research, Department of Clinical Science, University of Bergen, Bergen, Norway.

${ }^{2}$ Department of Pediatrics, Haukeland University Hospital, Bergen, Norway.

3 Medical and Population Genetics Program, Broad Institute of Harvard and MIT, Cambridge, MA ,USA.

${ }^{4}$ NORMENT Centre, Division of Mental Health and Addiction, Oslo University Hospital, Oslo, Norway.

5Institute of Clinical Medicine, University of Oslo, Oslo, Norway.

${ }^{6}$ Disease Systems Biology, Novo Nordisk Foundation Center for Protein Research, Faculty of Health and Medical Sciences, University of Copenhagen, Copenhagen, Denmark.

${ }^{7}$ Department of Biomedicine, Aarhus University, Aarhus, Denmark.

8iSEQ, Centre for Integrative Sequencing, Aarhus University, Aarhus, Denmark.

${ }^{9}$ iPSYCH, Lundbeck Foundation Initiative for Integrative Psychiatric Research, Aarhus, Denmark.

10Department of Laboratory Medicine, Karolinska Institutet, Stockholm, Sweden.

${ }^{11}$ Karolinska University Laboratory, Karolinska University Hospital, Stockholm, Sweden.

12The Estonian Genome Center, University of Tartu, Tartu, Estonia.

${ }^{14}$ Boston Children's Hospital, Harvard Medical School, Boston, MA, USA.

${ }^{14}$ Genetic and Molecular Epidemiology Unit, Lund University Diabetes Centre , Lund University , Malmö, Sweden.

${ }^{15}$ Department of Public Health and Clinical Medicine, Section for Medicine, Umeå University, Umeå, Sweden.

16Department of Nutrition, Harvard T. H. Chan School of Public Health, Boston, MA, USA.

${ }^{17}$ Radcliffe Department of Medicine, University of Oxford, Oxford, UK.

${ }^{18}$ Semel Institute for Neuroscience and Human Behavior, Los Angeles, CA, USA.

19UCLA Center for Neurobehavioral Genetics, Los Angeles, CA, USA. 
${ }^{20}$ FIMM Institute for Molecular Medicine Finland, University of Helsinki, Helsinki, Finland.

${ }^{21}$ School of Health and Medical Sciences, University of Copenhagen, Copenhagen, Denmark .

${ }^{22}$ Department for Congenital Disorders, Statens Serum Institut, Copenhagen, Denmark. ${ }^{23}$ iPSYCH - The Lundbeck Foundation Initiative for Psychiatric Research, Copenhagen, Denmark.

${ }^{24}$ Department of Tumor Biology, Institute for Cancer Research, Oslo University Hospital, Oslo, Norway.

${ }^{25}$ Institute of Cancer Genetics and informatics. Oslo University Hospital, Oslo, Norway. ${ }^{26}$ Department of Informatics, University of Oslo, Oslo, Norway.

${ }^{27}$ K.G. Jebsen Center for Genetic Epidemiology, Department of Public Health, Norwegian University of Science and Technology, Trondheim, Norway.

${ }^{28}$ HUNT Research Center, Department of Public Health, Norwegian University of Science and Technology, Levanger, Norway.

${ }^{29}$ Department of Medicine, Levanger Hospital, Levanger, Norway.

${ }^{30}$ Genomics and Biomarkers Unit, National Institute for Health and Welfare, Helsinki, Finland.

${ }^{31}$ Norwegian Institute of Public Health, Oslo, Norway.

${ }^{32}$ Department of Epidemiology Research, Statens Serum Institut, Copenhagen, Denmark. ${ }^{33}$ Department of Medicine, Stanford University School of Medicine, Stanford, CA, USA. ${ }^{34}$ Department of Clinical Medicine, University of Copenhagen, Copenhagen, Denmark. ${ }^{35}$ The Estonian Genome Center, University of Tartu, Tartu, Estonia.

${ }^{36}$ National Centre for Register-Based Research, Department of Economics and Business Economics, Aarhus University, Aarhus, Denmark.

37iPSYCH - The Lundbeck Foundation Initiative for Psychiatric Research, Aarhus, Denmark.

${ }^{38}$ Department of Medical Epidemiology and Biostatistics, Karolinska Institutet, Stockholm, Sweden.

${ }^{39}$ Swedish e-Science Research Center, SeRC, Stockholm, Sweden.

${ }^{40}$ Psychiatric and Neurodevelopmental Genetics Unit, Massachusetts General Hospital and Harvard Medical School, Boston, MA, USA.

41Stanley Center for Psychiatric Research, Broad Institute of MIT and Harvard, Cambridge, MA, USA.

${ }^{42}$ Analytic and Translational Genetics Unit, Massachusetts General Hospital and Harvard Medical School, Boston, MA, USA.

${ }^{43}$ Department of Neurology, Massachusetts General Hospital, Boston, MA, USA.

${ }^{44}$ Department of Research, Innovation and Education, Oslo University Hospital, Oslo, Norway. 
${ }^{45}$ deCODE genetics, Reykjavik, Iceland.

46University of Reykjavik, Iceland.

${ }^{47}$ Cardiovascular Division, Department of Medicine, Washington University, Saint Louis, MO, USA.

${ }^{48}$ Department of Genetics, Washington University, Saint Louis, MO, USA.

${ }^{49}$ McDonnell Genome Institute, Washington University, Saint Louis, MO, USA.

${ }^{50}$ Department of Medical Epidemiology and Biostatistics, Karolinska Institutet, Stockholm, Sweden.

${ }^{51}$ Department of Medical Biochemistry and Genetics, University of Turku, Turku, Finland.

${ }^{52}$ Institute of Biological Psychiatry, Mental Health Center Sct. Hans, Mental Health Services Capital Region of Denmark, Copenhagen, Denmark.

${ }^{53}$ Institute of Clinical Medicine, University of Copenhagen, Copenhagen, Denmark.

${ }^{54}$ Department of Global Public Health and Primary Care, University of Bergen, Bergen, Norway.

*Members of the writing group

Address correspondence to:

Professor Pål Rasmus Njølstad, M.D., Ph.D., Center for Diabetes Research, Hospital for Children and Adolescents, Haukeland University Hospital, Haukelandsbakken 15, NO5021 Bergen, Norway (pal.njolstad@uib.no).

\section{Contents}

Supplementary Table 1 


\begin{tabular}{|c|c|c|c|c|c|c|c|c|c|}
\hline & $\begin{array}{c}\text { Cardiovascular } \\
\text { disease }\end{array}$ & Schizophrenia & Type 2 diabetes & Lipid Disorders & Glioblastoma & $\begin{array}{c}\text { Alzheimer's } \\
\text { disease }\end{array}$ & Melanoma & All & \\
\hline & $n(\%)$ & $n(\%)$ & $n(\%)$ & $n(\%)$ & $n(\%)$ & $n(\%)$ & $n(\%)$ & $n(\%$ & \\
\hline Nordic & $93,510 \quad(48 \%)$ & $7,051 \quad(40 \%)$ & $9,817 \quad(35 \%)$ & $42,094 \quad(22 \%)$ & $4,067 \quad(13 \%)$ & 6,094 & $994 \quad(2 \%)$ & 163,627 & $(28 \%)$ \\
\hline USA & 7,018 & $2,281 \quad(13 \%)$ & $(13 \%)$ & 1,586 & 18,914 & 28,550 & $(21 \%)$ & 71,067 & $(12 \%)$ \\
\hline UK & $30,122 \quad(15 \%)$ & $4,407 \quad(25 \%)$ & $(25 \%)$ & 22,746 & $(13 \%)$ & 12,010 & 12,872 & 93,481 & $(16 \%)$ \\
\hline Other & 63,777 & $(22 \%)$ & $7,646 \quad(27 \%)$ & 122,151 & $(15 \%)$ & 27,089 & 20,390 & 249,792 & $(43 \%)$ \\
\hline Total & 194,427 & 17,709 & 28,350 & 188,577 & 31,954 & 73,743 & 43,207 & 577,967 & \\
\hline References & 23 & 24 & 25 & 26 & 27 & 28 & 29 & & \\
\hline
\end{tabular}

\section{Supplementary Table 1: Contribution of Nordic populations to published GWAS meta-analyses}

The extent to which patient samples are derived from the UK, USA, and Nordic countries for the major GWAS meta-analyses for seven diseases. Proportions were estimated from the European ancestry cohorts in each meta-analysis. In some rare instances, precise sample contributions could not be determined, as the contributing cohorts included participants from multiple countries; a best estimate was used in those cases based on the information provided in the supplementary materials and other cohort description papers. 
Governance and legislation

Unique personal identification number

Public health care systems

National registries that can be linked

Large population-based and patient cohorts with deep phenotypes and biological samples

Biobanks with large databases based on analyses of the biobank samples

Supplementary Table 2: Nordic resources to address global health challenges 


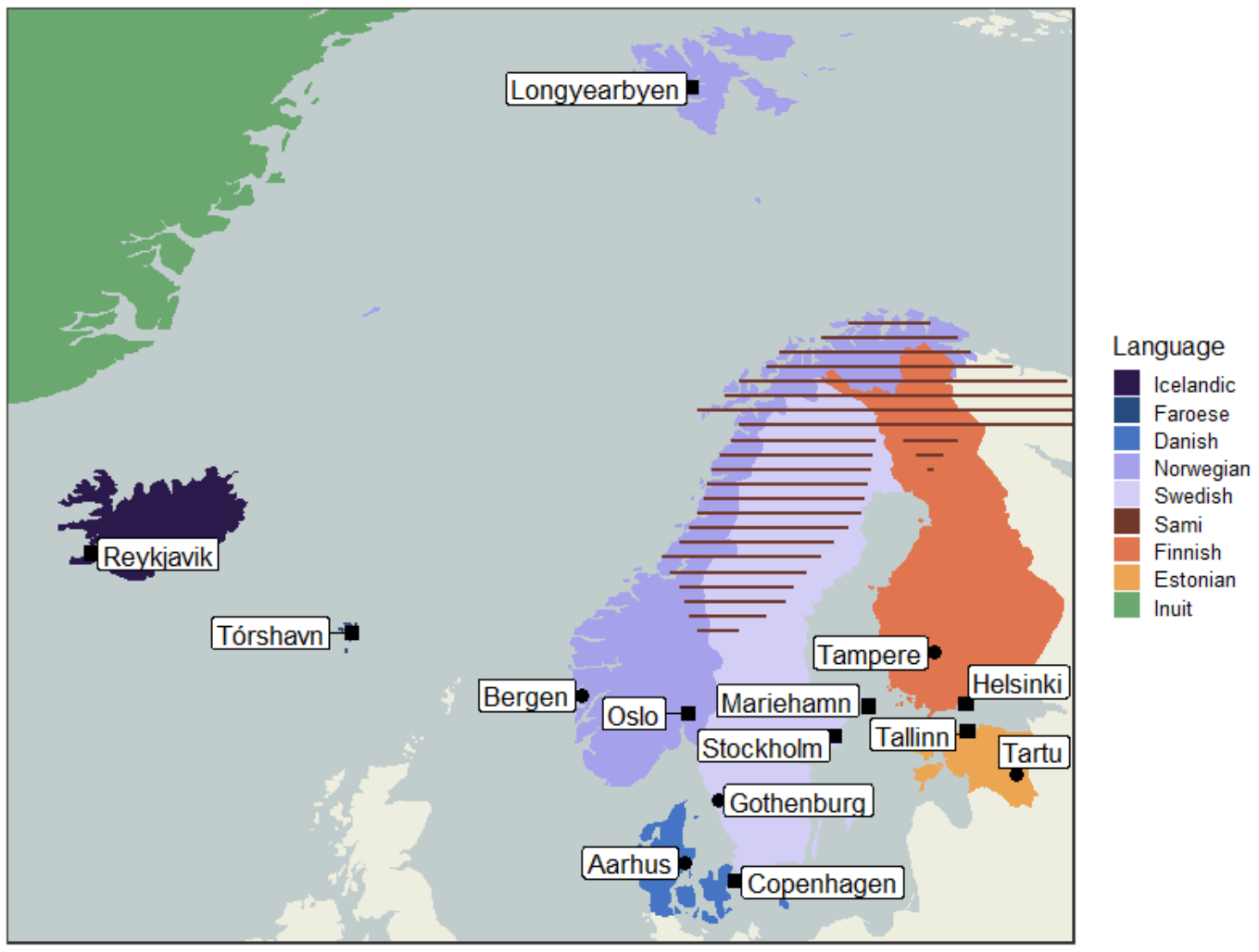

\section{Supplementary Figure 1: Languages of the Nordic region.}

The Nordic has three major linguistic families: 1) Danish, Faroese, Icelandic, Norwegian, and Swedish - rooted in the Old Norse language - belong to the North Germanic branch of the Indo-European languages and are displayed in shades of blue; 2) Finnish, Karelian, and Sami are part of the Finno-Ugric languages and displayed in a red to yellow gradient; 3) Inuit is a branch of the Eskimo-Aleut languages and is displayed in green. The capital of each country is shown by a filled square, while some the largest cities are shown by filled circles (Supplementary Note; European Commission, Joint Research Centre, Columbia University, NYC, NY). 


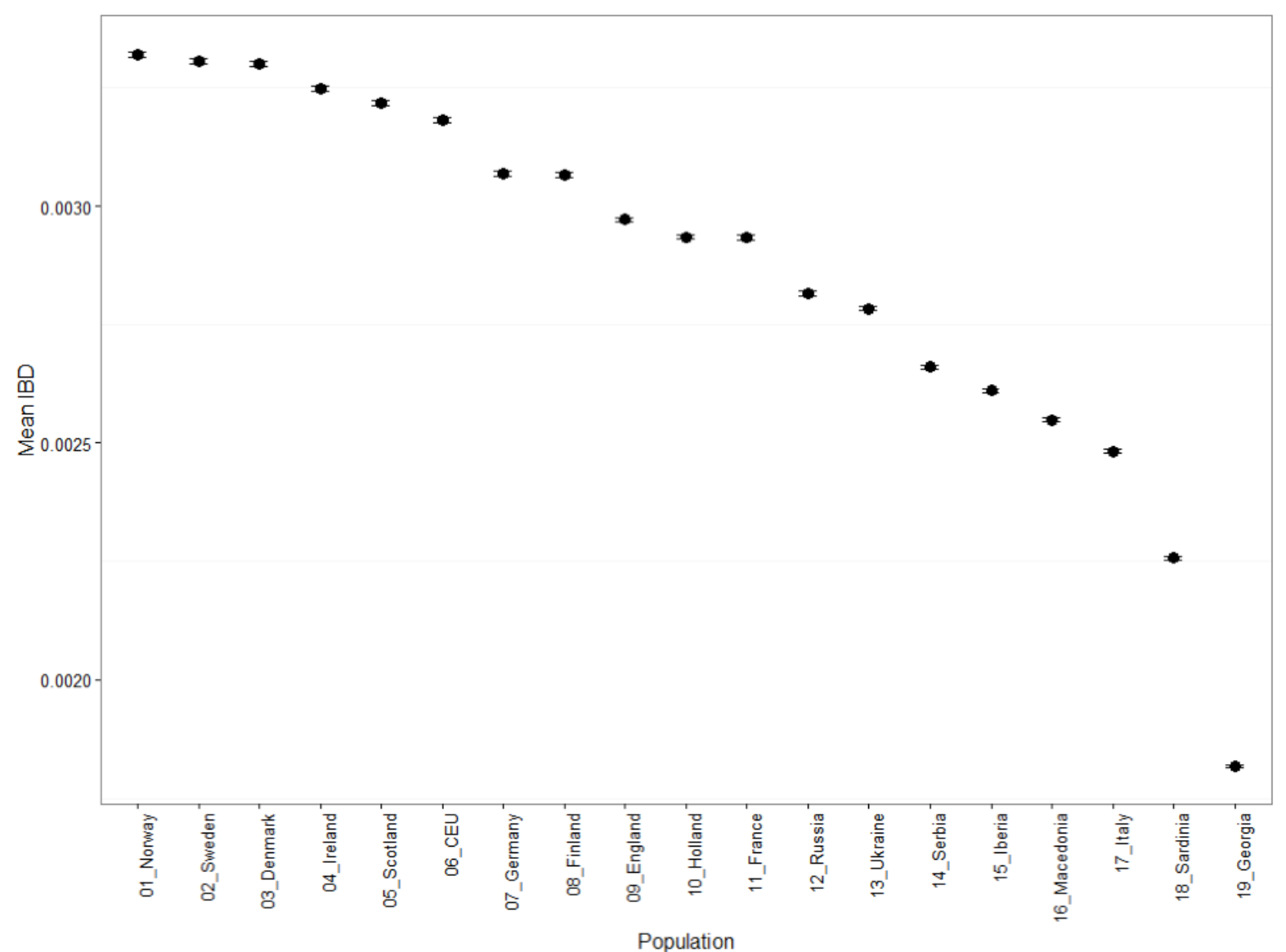

Supplementary Figure 2: Common variant sharing shows similarity of Nordic populations

A comparison of 22,500 Icelanders typed for the Illumina Omni Express SNP chip against 25 to 100 individuals with SNP chip data from each of 19 different populations of European ancestry - based on a common set of 144,248 SNPs. For each pair of individuals, we calculated the proportion of the genome shared in fragments longer than 2cM that are identical by descent (IBD). The figure shows the mean proportion of the genome shared IBD between the Icelanders and each of the 19 populations, with $95 \%$ confidence intervals. As expected, the greatest degree of sharing is with populations from Scandinavia and the British Isles. The fragments shared between Icelanders and these populations are longer because of more recent common ancestry, which implies a greater propensity to share rare mutations. 


\section{Supplementary Note: Method for generation of Figure 1 in the printed article}

Figure 1 of the published article: This map was generated using a script provided in the link below. In short, the world map using the Mollweide projection was plotted in light yellow over a light blue background. The countries of the Nordic region, composed here of Estonia, Finland, Denmark, Iceland, Norway, and Sweden, and associated territories, the Faroe Islands, Greenland, Svalbard, and the Åland Islands, were plotted in different colors and outlined in black.

Population density as of 2015 was obtained from the Global Human Settlement project at a resolution of $1 \mathrm{~km}$ (European Commission, Joint Research Centre [JRC]; Columbia University, Center for International Earth Science Information Network - CIESIN [2015]: GHS population grid, derived from GPW4, multitemporal [1975, 1990, 2000, 2015]. European Commission, Joint Research Centre [JRC]).

The density matrix was rasterized in 2000 bins in latitude and longitude over the Nordic region, aligned onto the world map, and displayed in shades of grey. Finally, the name along with the number of inhabitants was annotated for each country and associated territory. The number of inhabitants was the latest available in Wikipedia at time of writing.

Supplementary Figure 1: This map was generated using the script in the link below. In short, the world map using the Mollweide projection is plotted in light yellow over a light blue background as in the population map.

The Nordic countries and associated territories were colored according to the official language. The Sami language was annotated with horizontal segments. Capitals and major cities were annotated with points and names using their latitude and longitude as obtained from latlong.net. North Germanic languages were annotated using a gradient of blue, Finno-Ugric languages using a red to yellow gradient, and Inuit as part of EskimoAleut languages in green.

\section{URLS:}

Script: https://github.com/mvaudel/Nordic-maps Global Human Settlement project: https://ghsl.jrc.ec.europa.eu/ghs pop.php European Commission, Joint Research Centre (JRC): http://data.europa.eu/89h/jrc-ghslghs pop gpw4 globe r2015a) 